\title{
INT monitoring survey of Local Group dwarf galaxies: star formation history and chemical enrichment
}

\author{
T. Parto ${ }^{* 1,2}$, Sh. Dehghani ${ }^{3}$, A. Javadi ${ }^{1,4}$, E. Saremi ${ }^{1}$, J. Th. van Loon ${ }^{5}$, H. Khosroshahi ${ }^{1}$, M. $^{2}$ \\ T. Mirtorabi ${ }^{2}$, H. Abdollahi ${ }^{2}$, M. Gholami ${ }^{2}$, S.A. Hashemi ${ }^{4}$, M. Navabi ${ }^{1}$, M. Noori ${ }^{6}$, S. T. \\ Aghdam $^{1}$, M. Torki ${ }^{6}$, and M. Vafaeizade ${ }^{2}$ \\ ${ }^{1}$ School of Astronomy, Institute for Research in Fundamental Sciences (IPM), PO Box 19395-5531, Tehran, Iran \\ ${ }^{2}$ Physics Department, Alzahra University, Vanak, 1993891176, Tehran, Iran \\ ${ }^{3}$ Physik. Institut, University of Cologne, Zulpicher Str. 77, D-50937 Cologne, Germany \\ ${ }^{4}$ Department of Physics, Sharif University of Technology, Tehran, 11155-9161, Iran \\ ${ }^{5}$ Astrophysics Group, Lennard-Jones Laboratories, Keele University, Staffordshire ST5 5BG \\ ${ }^{6}$ Physics Department, Faculty of Science, University of Zanjan, Zanjan 45371-38791, Iran
}

\begin{abstract}
The Local Group (LG) hosts many dwarf galaxies with diverse physical characteristics in terms of morphology, mass, star formation, and metallicity. To this end, LG can offer a unique site to tackle questions about the formation and evolution of galaxies by providing detailed information. While large telescopes are often the first choices for such studies, small telescope surveys that perform dedicated observations are still important, particularly in studying bright objects in the nearby universe. In this regard, we conducted a nine epoch survey of 55 dwarf galaxies called the "Local Group dwarf galaxies survey" using the $2.5 \mathrm{~m}$ Isaac Newton Telescope (INT) in the La Palma to identify Long-Period Variable (LPV) stars, namely Asymptotic Giant Branch (AGB) and Red Super Giant (RSG) stars. AGB stars formed at different times and studying their radial distribution and mass-loss rate can shed light on the structure formation in galaxies. To further investigate the evolutionary path of these galaxies, we construct their star formation history (SFH) using the LPV stars, which are at the final stages of their evolution and therefore experience brightness fluctuations on the timescales between hundred to thousand days. In this paper, we present some of the results of the Local Group dwarf galaxies survey.
\end{abstract}

Keywords: stars: $A G B$ and RSG - Stars: LPVs - Stars: dust - galaxies: evolution - galaxies: star-formation - galaxies: Local-Group - galaxies: dwarf

\section{Introduction}

Dwarf galaxies are the most common type of galaxies in the universe. The importance of the internal and external processes (e.g., supernova explosion; interaction with the massive halos) on the evolution of these small galaxies are well known, though with many unanswered aspects (Weisz et al. 2014; Saremi et al. 2020). The star formation history (SFH) is a robust tracer of how different internal or external mechanisms affect the evolution of a galaxy (Saremi et al. 2019b).

The cool asymptotic giant branch (AGB) stars with luminosities of $\sim 10^{4} L_{\odot}$ and wide age ranges, from 100 Myr to older than 10 Gry, are well-known probes of the stellar population of galaxies in the near-infrared (Habing et al. 2004; Javadi et al. 2011a, 2017)

especially in the nearby universe. Many evolved AGBs, to be specific, thermally pulsing AGBs (TP-AGB), are long-period variables (LPVs; Marigo et al. 2017), therefore they experience brightness fluctuations on the timescales between 100 to 1300 days due to the low surface gravity (Javadi \& van Loon 2019). TP-AGBs are responsible for a significant fraction of the integrated light of a galaxy, they also contribute meaningfully to the chemical enrichment of the interstellar medium (ISM) through

*tahereparto@gmail.com, Corresponding author 
different mechanisms that drive the stellar wind. Red supergiant (RSG) stars with the look-back time $10^{7}$ years are another major dust producers and are examples of LPVs and their brightness variates between 600 to 900 days (Javadi \& van Loon 2019).

This survey aims to uniformly determine the SFH of 55 dwarf galaxies of the Local Group (LG) by adopting a novel method. This method was first proposed and applied to the M33 by (Javadi et al. 2011b) and relies on the identification of LPVs. There are some other successful applications of this method on dwarf galaxies in LG (e.g., IC 1613 Hashemi et al. 2018; LMC and SMC Rezaeikh et al. 2014; NGC 147 and NGC 185 Hamedani Golshan et al. 2017). Using this method, We can also monitor the amount of produced dust by LPVs, and investigate their role in the star formation and evolution of a galaxy. Surveying this large sample of LG dwarfs, enables us to determine the evolutionary dependense of dwarfs galaxies on the enviroment, such as proximity to the host galaxies and compare it with internal effects like the stellar mass or gas content (Saremi et al. 2019a).

\section{Observations and Data}

The sample of dwarf galaxies studied in this survey consists of all observable Andromeda system of satellites in the northern hemisphere, along with 20 satellites of Milky Way and some isolated and transitional dwarfs (Saremi et al. 2017). We exclude galaxies that have been studied with Javadi's method before.

The Observations were made in 9 epochs between 2015-2018 using the Wide Field Camera (WFC), an optical mosaic camera on the INT telescope. WFC consists of four $2048 \times 4096$ CCDs, with a pixel scale of $0.33 \mathrm{arcsec} / \mathrm{pixel}$.

We used the i-band filter that is suited for identifying dusty AGBs. We also performed observations in the V-band to obtain the changes in the color (temperature) of stars. For each galaxy, the observation nights are separated by a month or more to identify LPV stars.

We reduce the WFC images using the THELI image processing pipeline (Erben et al. 2005). After the reduction process, We perform photometry for the crowded-field using DAOPHOT II (Stetson 1987) to construct a catalog for each galaxy. Furthermore, a completeness test carried out using the ADDSTAR task in the DAOPHOT II package to show the depth of our photometry. To remove the foreground contamination, we impose selection criteria on the proper motion and parallax of stars estimated in the GAIA Data Release 2 (Gaia Collaboration et al. 2018). The complete description of observations and details of the photometry procedure is available at (Saremi et al. 2020).

\section{Method}

\subsection{LPV candidates detection}

To identify LPV candidates, we employed a method described in (Stetson 1996) to determine the variability index $\mathrm{L}$ for each star. Then we estimated a threshold for variability, using the variability distribution for the stars in different magnitude bins (Javadi et al. 2011a; Saremi et al. 2020)

With insufficient observation night, we can not obtain a meaningful period for LPVs. However, there is a correlation between the amplitude and period of a variable star (Goldman et al. 2019), and large amplitude variables (LAV) are usually evolved AGBs at the late stage of their life.

Fig. 1 shows the color-magnitude diagram (CMD) of SagDIG, one of the dwarf irregular galaxies in our sample with the overplotted PARSEC-COLIBRI isochrones (Marigo et al. 2017). LPV candidates are shown as blue circles, with a size scaled to their amplitude in the i-band with values between $0.1-1.87 \mathrm{mag}$.

\subsection{SFH from LPV pulsation}

The luminosity of AGB stars reaches a maximum at the final stage of life, hence can be used to estimate the birth mass of the star. For determining the mass, we construct the mass function of LPVs (for the suitable metalicity) by interpolating mass-luminosity relation using the PARSEC-COLIBRI isochrones. After estimating age of LPVs using the mass-age relation, we determine pulsation duration 
by fitting multiple Gaussian functions to the mass-pulsation values in the isochrones that show strong pulsations.

The star formation rate (SFR) function $\xi(t)$ introduced in (Javadi et al. 2011b) takes mass, age and pulsation duration of LPVs and estimates the stellar mass formed per year $\left(M_{\odot} y^{-1}\right)$ :

$$
\xi(t)=\frac{\mathrm{dn}(\mathrm{t})}{\delta t} \frac{\int_{\min }^{\max } f_{I M F}(m) m d m}{\int_{m(t)}^{m(t+d t)} f_{I M F}(m) m d m} .
$$

where $f_{I M F}$ is the initial mass function (Kroupa 2001), $d t$ represents different age bins, and $d n$ is the number of observed LPVs in each age bins.

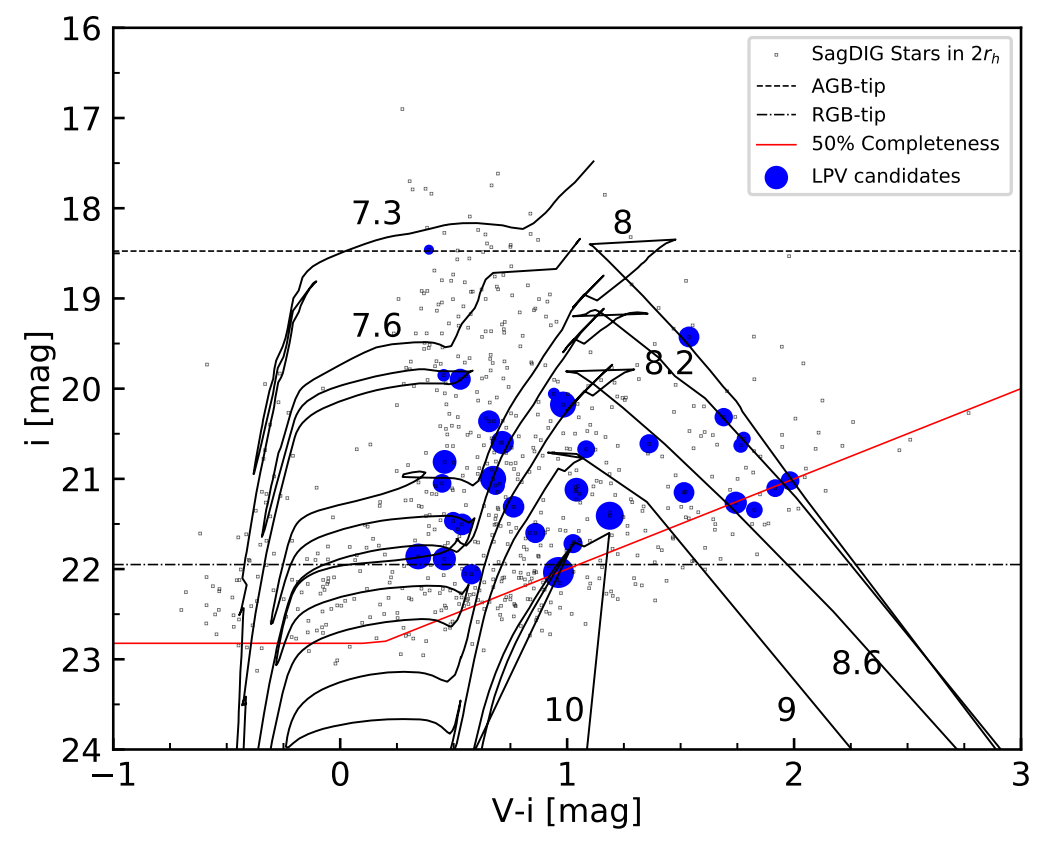

Figure 1. [i] vs. [V-i] CMD for SagDIG with the overplotted isochrones. Black dots denote the stars within the two half-light radius of this galaxy. Blue circles are the LPV candidates with a size scaled to their amplitude. The dotted line marks RGB-tip and AGB-tip. The 50\% completeness is represented by the solid red line.

\section{Results and On-going works}

Here we present results of published papers for three galaxies: And I and And VII, two dwarf spheroidal and satellite of Andromeda, and IC 10, an irregular and isolated galaxy.

(Saremi et al. 2020) detected 59 LPV candidates within the two half-light radii of And I, including five extreme AGBs (X-AGB). They also modeled the spectral energy distribution (SED) of these stars, using DUSTY code (Ivezic \& Elitzur 1997) and mid-IR bands measurement from Spitzer (3.6 and 4.5 $\mu m$ ), WISE ( $\mathrm{W} 1=3.4, \mathrm{~W} 2=4.6, \mathrm{~W} 3=11.6$, and $\mathrm{W} 4=22 \mu \mathrm{m})$ (Cutri \& et al. 2014; Wright et al. 2010), INT near-infrared i-band catalog and SDSS $(\mathrm{u}, \mathrm{g}, \mathrm{r}, \mathrm{z})$ filters. They evaluated the total mass-loss rate of $3.5 \times 10^{-5} M_{\odot} y r^{-1}$ from five X-AGBs and thirteen dusty AGBs, which suggest low growth of stellar mass $(\sim 10 \%)$ in AND I in the next 10 Gyr.

(Navabi et al. Submitted, 2020) detected 43 LPV candidates within the half-light radii of And VII, and estimated the SFR peak of this area about $0.002 M_{\odot} y r^{-1}$ at $\mathrm{Z}=0.0007$. Probably, And VII was quenched by environmental impacts after infall into Andromeda's virial radius (Navabi et al. 2020a, 2020b). 
(Gholami et al. 2019) detected around 10000 AGB stars in IC 10 in the area of CCD4 $(\sim 0.07$ square-degree). They found that the AGB population concentrated more in the central region of IC 10, while the red giant branch (RGB) and RSG stars are more spread (Gholami \& Mirtorabi 2019).

In the following, we complete this study for other dwarfs and construct the dust map of the galaxy. We later discuss our results in light of different structure formation scenarios and the importance of internal feedbacks in LG dwarf galaxies.

\section{Acknowledgements}

The observing time for this survey is provided by the Iranian National Observatory and the UK-PATT allocation of time to programs I/2016B/09 and I/2017B/04 (PI: J. van Loon). The authors thank the Iranian National Observatory and the School of Astronomy (IPM) for the financial support of this project.

\section{References}

Cutri R. M., et al. 2014, VizieR Online Data Catalog, p. II/328

Erben T., et al., 2005, AN, 326, 432

Gaia Collaboration et al., 2018, A\&A, 616, A1

Gholami M., Mirtorabi M., 2019, IJAA, 6, 53

Gholami M., Javadi A., van Loon J. T., Khosroshahi H., Mirtorabi M. T., Saremi E., Eftekhari S., 2019, in McQuinn K. B. W., Stierwalt S., eds, Vol. 344, Dwarf Galaxies: From the Deep Universe to the Present. pp 70-72, doi:10.1017/S1743921318006531

Goldman S. R., et al., 2019, ApJ, 877, 49

Habing H., Olofsson H., Editors. 2004, Asymptotic Giant Branch Stars. Springer

Hamedani Golshan R., Javadi A., van Loon J. T., Khosroshahi H., Saremi E., 2017, MNRS, 466, 1764

Hashemi S. A., Javadi A., van Loon J. T., 2018, MNRAS, 483, 4751

Ivezic Z., Elitzur M., 1997, MNRAS, 287, 799

Javadi A., van Loon J. T., 2019, IAU Symposium, 343, 283

Javadi A., van Loon J. T., Mirtorabi M. T., 2011a, MNRS, 411, 263

Javadi A., van Loon J. T., Mirtorabi M. T., 2011b, MNRAS, 414, 3394

Javadi A., van Loon J. T., Khosroshahi H. G., Tabatabaei F., Hamedani Golshan R., Rashidi M., 2017, MNRAS, 464, 2103

Kroupa P., 2001, MNRAS, 322, 231

Marigo P., et al., 2017, ApJ, 835, 77

Navabi M., Noori M., Saremi E., Javadi A., van Loon J., Khosroshahi H., 2020a, in Neiner C., Weiss W. W., Baade D., Griffin R. E., Lovekin C. C., Moffat A. F. J., eds, Stars and their Variability Observed from Space. pp 383-385

Navabi M., Saremi E., Javadi A., Noori M., van Loon J., Khosroshahi H., 2020b, Proceedings of 27th Ipm Physics Spring Conference, 20

Rezaeikh S., Javadi A., Khosroshahi H., van Loon J. T., 2014, MNRAS, 445, 2214

Saremi E., et al., 2017, Journal of Physics: Conference Series, 869, 012068

Saremi E., Javadi A., van Loon J., Khosroshahi H., Torki M., 2019a, in Griffin R. E., ed., Vol. 339, Southern Horizons in TimeDomain Astronomy. pp 336-339, doi:10.1017/S1743921318002958

Saremi E., Javadi A., van Loon J. T., Khosroshahi H., Rezaei Kh S., Golshan R. H., Hashemi S. A., 2019b, in McQuinn K. B. W., Stierwalt S., eds, Vol. 344, Dwarf Galaxies: From the Deep Universe to the Present. pp 125-129 (arXiv:1812.09725), doi:10.1017/S1743921318005574

Saremi E., et al., 2020, Apj, 894, 135

Stetson P. B., 1987, PASP, 99, 191

Stetson P. B., 1996, PASP, 108, 851

Weisz D. R., Dolphin A. E., Skillman E. D., Holtzman J., Gilbert K. M., Dalcanton J. J., Williams B. F., 2014, ApJ, 789, 147

Wright E. L., et al., 2010, AJ, 140, 1868 